\title{
A catalog of LEDA galaxies with DENIS measurements ${ }^{\star}$
}

\author{
G. Paturel ${ }^{1}$, I. Vauglin ${ }^{1}$, C. Petit ${ }^{1}$, J. Borsenberger ${ }^{2}$, N. Epchtein ${ }^{3}$, P. Fouqué ${ }^{4}$, and G. Mamon ${ }^{5}$ \\ ${ }^{1}$ CRAL - Observatoire de Lyon, 69561 Saint-Genis Laval Cedex, France \\ e-mail: patu@obs.univ-lyon1.fr \\ 2 Observatoire de Paris-Meudon, 5, place Jules-Janssen, 92195 Meudon, France \\ ${ }^{3}$ LUAN, Université de Nice Sophia-Antipolis, CNRS - UMR 6525, Parc Valrose, Nice, France \\ ${ }^{4}$ Observatoire Midi-Pyrénées, 14 avenue Edouard-Belin, 31400 Toulouse, France \\ 5 Institut d'Astrophysique de Paris, 98B Bd. Arago, 75014 Paris, France
}

Received 26 April 2004 / Accepted 17 September 2004

\begin{abstract}
We present a catalog of near-infrared properties of LEDA galaxies, using the full resolution images from the DENIS survey. The fluxes are integrated in eight homothetic ellipses defined by their proper axis ratio, position angle and major axis (up to twice the blue diameter at the isophote $25 \mathrm{mag} \mathrm{arcsec}^{-2}$ ) extracted from the LEDA database. From the curves of growth in $I, J$ and $K_{\mathrm{s}}$ photometric bands, we estimated different apparent magnitudes and diameters ("total", "Kron" and "isophotal"). Isophotal parameters refer to the limiting surface brightnesses: $22.5 \mathrm{I} \mathrm{mag} \operatorname{arcsec}^{-2}, 21.0 \mathrm{~J} \mathrm{mag}^{-\operatorname{arcsec}^{-2}}$ and $20.0 K_{\mathrm{s}} \mathrm{mag} \operatorname{arcsec}^{-2}$ for the three photometric bands, respectively. The result is a catalog of 753153 objects (among which there are 508224 galaxies, 34449 probable galaxies and 210480 galaxies to be confirmed). The catalog gives about (the figures vary, depending on the considered magnitude or diameter): $668000 \mathrm{I}$-band magnitudes, $576000 \mathrm{~J}$-band magnitudes, $357000 K_{\mathrm{s}}$-band magnitudes and $452000 \mathrm{I}$-band diameters, $299000 \mathrm{~J}$-band diameters, $114000 \mathrm{~K}_{\mathrm{s}}$-band diameters.

The typical standard deviations for $I, J$ and $K_{\mathrm{s}}$ magnitudes are $0.14,0.15$ and 0.25 , respectively, for magnitudes limited at $I=16, J=15$ and $K_{\mathrm{s}}=14$. The contamination by superimposed objects probably remains the major source of problems and could require future improvement. The completeness limits in magnitude are about: 15.5, 14.5 and 13 in $I, J$ and $K_{\mathrm{s}}$, respectively.
\end{abstract}

Key words. galaxies: general - catalogs

\section{Introduction}

DENIS was the first attempt to carry out a very large near infrared survey from a direct electronic acquisition on the sky. The project was first presented by Epchtein (1997). The result of seven years of measurements, conducted under the control of P. Fouqué, has lead to an impressive amount of data: about 900000 images in Gunn- $I$ band at $0.8 \mu \mathrm{m}$, in $J$ band at $1.25 \mu \mathrm{m}$ and $K_{\mathrm{s}}$ band at $2.12 \mu \mathrm{m}$. Each elementary image is $12^{\prime} \times 12^{\prime}$ with a pixel size of $1^{\prime \prime}$ (for $J$ and $K_{\mathrm{s}}$ this spatial resolution is obtained by microscanning). The integration time is $10 \mathrm{~s}$. The sequence of observation is made at a given right ascension for a wide range of declination $\left(30^{\circ}\right)$. This arrangement is called a strip. It contains 180 elementary images with $1^{\prime}$ overlap on each side.

Today many such large surveys have been undertaken in different wavelength bands (2MASS, SLOAN, HIPASS, FIRST). The 2MASS project was conducted on the whole

* Full Catalog illustrated in Table 6 is only available in electronic form at CDS via anonymous ftp to cdsarc.u-strasbg.fr $(130.79 .128 .5)$ or via

http://cdsweb.u-strasbg.fr/cgi-bin/qcat?J/A+A/430/751 sky with similar purposes: a very large sky infrared survey. It uses $J, H$ and $K_{\mathrm{s}}$ bands (Jarrett et al. 2000) with two dedicated telescopes. DENIS was conducted in a more difficult way, using a telescope rented to ESO. For the extragalactic domain we produced preliminary catalogs using low resolution images (Vauglin et al. 1999; Paturel et al. 2003b; Rousseau et al. 2000; Vauglin et al. 2002).

In this paper we present an exploitation of the DENIS survey using full resolution images. This leads to a catalog of 753153 galaxies with $I, J, K_{\mathrm{s}}$ magnitudes. This is still a provisional catalog because the final DENIS catalog (Mamon et al. 2004, in preparation) will not be restricted to LEDA objects ${ }^{1}$. It will be deeper, more accurate and more homogeneous. Nevertheless, the present catalog should be useful for preliminary studies, especially when $I$-band data is required. The present catalog is about ten times larger than the one we

${ }^{1} 85 \%$ of galaxies in LEDA come from the automatic analysis of the POSS1 survey (Paturel et al. 2000) and 15\% come from the literature, the main contributions coming from: Vorontsov-Velyaminov et al. (MCG), Nilson (UGC), Lauberts \& Valentijn (ESO and ESOLV), Zwicky et al. (CGCG) - see Paturel et al. (2003a) for the details. 


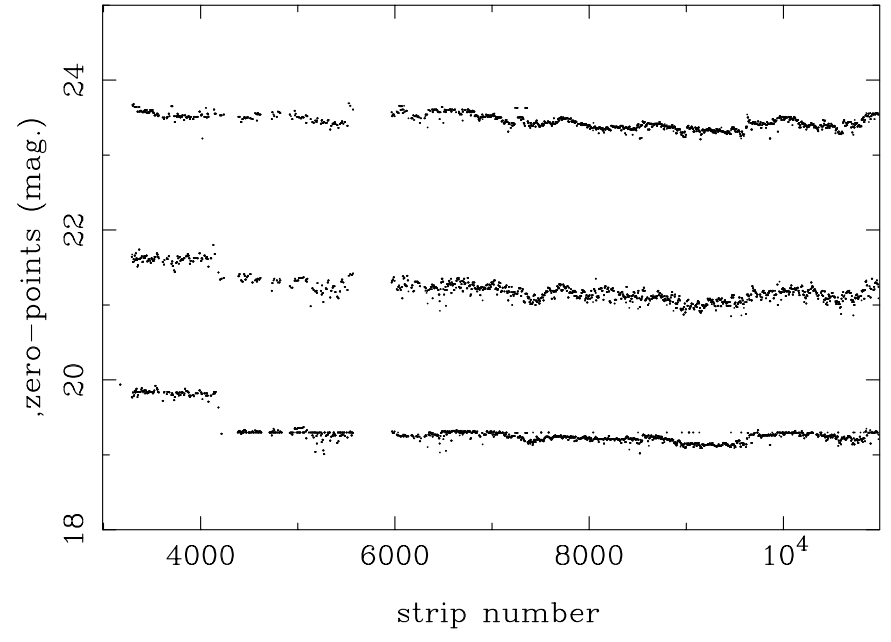

Fig. 1. Variation of adopted zero-points with strip number (i.e. with time) for $I, J$ and $K_{\mathrm{s}}$ photometric bands.

Table 1. Statistics of the different flags, for $I, J$ and $K_{\mathrm{s}}$ photometric bands.

\begin{tabular}{lrrr}
\hline \hline Qualityflag & $I$ & $J$ & $K_{\mathrm{s}}$ \\
\hline$f_{\text {zp }}$ & $8 \%$ & $9 \%$ & $8 \%$ \\
$f_{\text {qlt }}$ & $12 \%$ & $11 \%$ & $12 \%$ \\
$f_{\text {edge }}$ & $1 \%$ & $1 \%$ & $2 \%$ \\
$f_{*}$ & $18 \%$ & $25 \%$ & $37 \%$ \\
\hline
\end{tabular}

produced, for $I$-band only, with low resolution images (Paturel et al. 2003b).

\section{First reduction process and checking}

The data reduction was conducted by Borsenberger (1997). The data received from the observational site of La Silla are first sent to the Paris Data Analysis Center where they are preprocessed. They are flat-fielded and the sky background is subtracted. The original coordinate system given in the header is replaced by a calibrated one obtained by a cross-identification of stellar sources with the USNO-A2.0 astrometric catalog (Monet et al. 1998). Despite this improvement some coordinates are still uncertain. We will see below how this problem is solved.

Many primary or secondary photometric standards were measured at least twice every 180 images (strip). From this analysis one of us (JB) derived the $I, J$ and $K_{\mathrm{s}}$ zero points needed to transform intensities to absolute fluxes. The adopted zero points are given for each night (the strips from the same night have thus the same zero-point). The variations along the survey are shown in Fig. 1. Some abrupt changes correspond to cleaning of mirrors or modifications of the camera. When it was not possible to measure zero-points, we adopt the mean between the previous and next values, instead of using a default value. In such cases a flag is put on the corresponding data. This flag is designated as $f_{\mathrm{zp}}$. The statistics of flagged objects is given in Table 1 .

During the DENIS survey there was no automatic monitoring to check the quality of the sky. So, we use the stability of

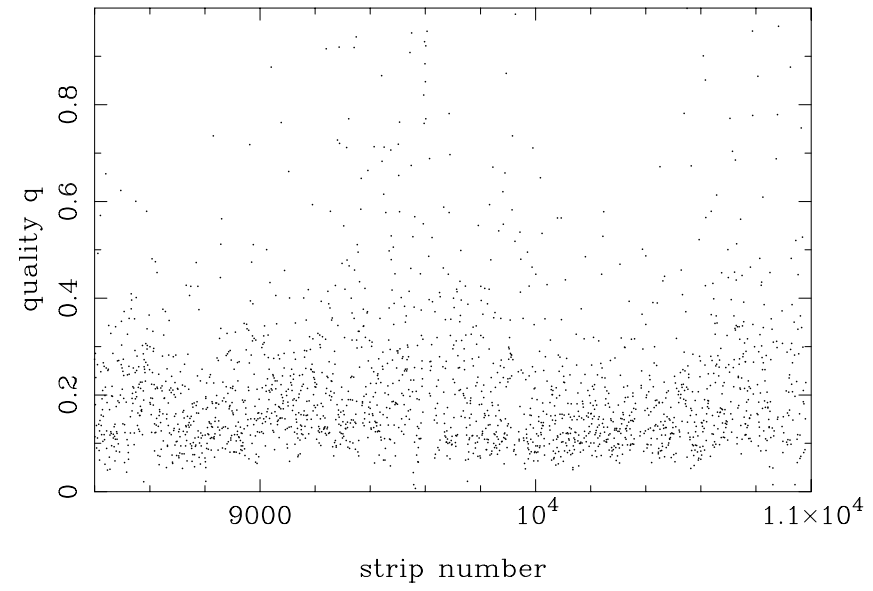

Fig. 2. Variation of quality estimated from the fluctuations of the sky background along a strip as a function of strip number for the $I$ photometric band.

the sky background to test the reliability as showed in a previous paper (Vauglin et al. 2002). The sky background may vary along a strip, e.g. when the strip crosses the Milky Way. However, the standard deviation of the sky background calculated for an elementary image should be more stable along a strip if the sky is clear. On the contrary, if clouds cross the field, the pixel intensities will vary at random and the standard deviation of the sky background will increase. Of course, one cannot exclude that unresolved stars also affect the standard deviation of the sky background in crowed fields, but this will also produce poorer data ${ }^{2}$. Thus, the fluctuations of the standard deviation of the sky background along a strip is used to estimate the quality $q$ of the strip. The definition is thus:

$q=\sigma_{\text {strip }}\left(\sigma_{\text {image }}(\right.$ skybackground $\left.)\right)$,

where $\sigma_{\text {domain }}$ (quantity) is the standard deviation of "quantity" calculated over "domain". As an example we show the quality $q$ for the $I$-band for all strips of the survey (Fig. 2). When $q$ is higher than $0.3 \mathrm{mag}$ (in $I$ ) or $0.2 \mathrm{mag}$ (in $J$ or $K_{\mathrm{s}}$ ) a flag is put on the measurement. This flag is designated as $f_{\text {qlt }}$. The statistics of flagged objects are given in Table 1.

\section{Recognition and photometry of galaxies}

Our previous experience (Vauglin et al. 1999; Paturel et al. 2000) showed us that it is difficult to have full reliability with automatic recognition of galaxies. So, we made use of our LEDA database containing about 2 million galaxies from which about 1 million galaxies have been confirmed either by redshift measurements or by visual inspection up to the 18th $B$-magnitude. By selecting objects in the DENIS survey area, 921340 galaxies are kept (of which 565000 are considered as confirmed galaxies).

Further, because the coordinates in LEDA are relatively good (the standard deviation is typically a few arcseconds

\footnotetext{
${ }^{2}$ We checked also some possible correlations (e.g., $q$ vs. $\sec \zeta$ or $q$ vs. $|b|)$ without finding any significant correlation. The quality of images reflects only the quality of the sky, at the time of observation.
} 


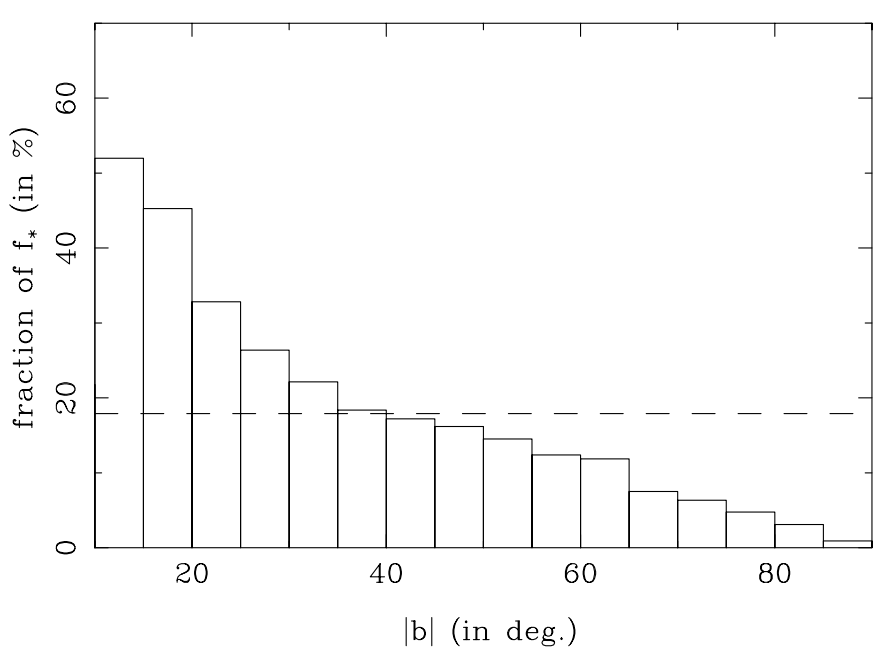

Fig. 3. Fraction of objects having a flag $f_{*}$ as a function of absolute value of the galactic latitude. The dashed line represents the mean fraction.

according to Paturel et al. 1999) this solves the remaining problem of DENIS coordinates. We will keep only objects for which a good agreement exists between both coordinate determinations.

From LEDA we also extracted the diameter $D_{25}$, axis ratio $R_{25}$ and position angle $\beta$ for all selected galaxies. The dimensions refer, on average, to the blue limiting surface brightness $25 \mathrm{mag} \operatorname{arcsec}^{-2}$. Diameters and position angle are used to integrate the flux in eight homothetic ellipses having diameters regularly distributed between $D_{25} / 4$ and $2 D_{25}$ (i.e., $2 D_{25} / i$, $i$ down from eight to one).

During this integration process we searched for the maximum of the pixel intensity within the ellipse of diameter $2 D_{25} / 5$. This defines the adopted center of the galaxy. The central flux and the surface area of the corresponding ellipse is used to estimate the object type (star or galaxy) when it is not confirmed from LEDA (see Sect. 4). We calculate the DENIS coordinates for the pixel of maximum central intensity. When the discrepancy with the LEDA coordinates exceeds twice the minor axis or is greater than $30^{\prime \prime}$, a flag is put on the measurement. Objects having a problem of coordinates are rare (a few percent). They are simply removed.

In order to remove superimposed stars we use the following procedure: when a pixel intensity is higher than the maximum found at the center of the galaxy, we replace the surface area corresponding to the Point Spread Function (PSF) around this pixel by a constant intensity. This constant intensity is chosen in the vicinity of the considered pixel (typically at a distance corresponding to the PSF radius). When the total correction exceeds 0.3 mag a flag is put on the measurement. This flag will be designated as $f_{*}$. The statistics of flagged objects are given in Table 1. As expected, the number of objects that have this flag increases towards low galactic latitude (Fig. 3). Note that the presence of a flag does not mean that the magnitude should be rejected. It simply means that a correction for superimposed stars has been applied.

The eight fluxes within the eight homothetic ellipses are transformed into magnitudes using proper zero points (previous

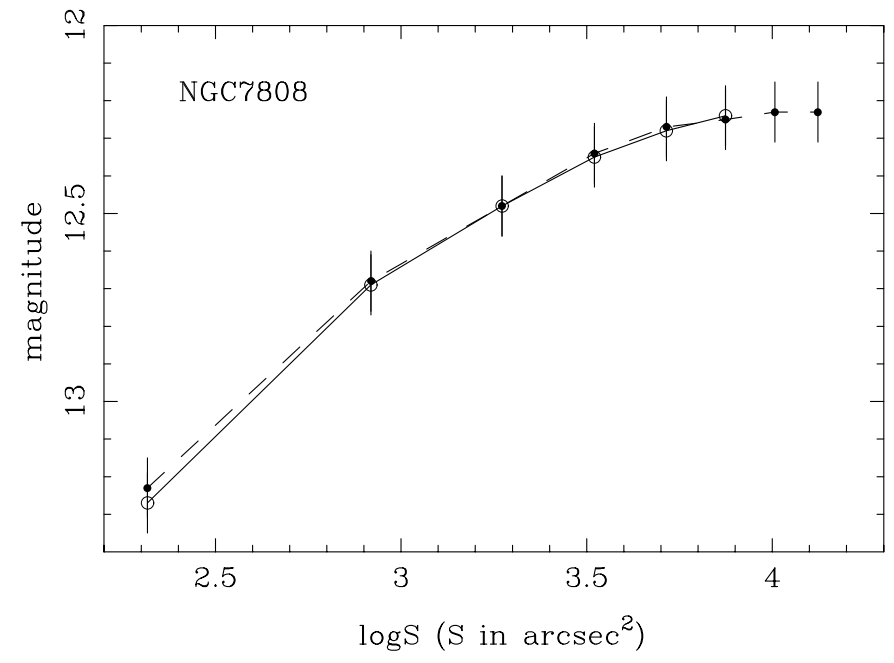

Fig. 4. The curve of growth of a typical galaxy (NGC 7808). The points (filled circles) and dashed line represent the raw measurements obtained by integrating within the eight homothetic ellipses defined in the text. The open circles and solid line represent the measurements corrected for the local sky-background calculated from the two largest ellipses. The error bars are obtained from the standard deviation of the curve of growth.

section) and constant atmospheric extinction coefficients: 0.02 in $I, 0.08$ in $J$ and 0.11 in $K_{\mathrm{s}}$. Because the sky background has been reduced to zero, on average, the magnitudes do not need, in principle, a sky background subtraction. In practice, the local sky background is estimated from external ellipses. We give an example of a curve of growth (magnitude vs. surface area) for a typical galaxy arbitrarily chosen (Fig. 4). In the next section, we use the surface brightness profile for each galaxy.

The final test of quality is made by flagging galaxies that are near the edge of the frame. If the marginal projections of the largest ellipse have more than five percent length outside the limit of the frame, a flag $f_{\text {edge }}$ is put on the measurements for this object. Table 1 summarizes the statistics of all flags.

After this first step we obtain three catalogs. They contain: 1562677 measurements for 724973 objects in $I$, 1315302 measurements for 633049 objects in $J$ and 934816 measurements for 416234 objects in $K_{\mathrm{s}}$. Many objects are measured several times because of the overlap of the images along a strip and the overlap of adjacent strips. Thus, we have to calculate the mean curve of growth for each object.

\section{Mean of different determinations}

To calculate the weighted mean of different measurements we have first to estimate the standard deviation of each of them. Because the standard error on magnitudes is the same as the standard error on surface brightness (assuming there is no uncertainty on the calculation on the surface area), this standard error is estimated through a fit of a parametric Sersic profile (surface brightness $S B$ vs. $r^{1 / n}$, as shown for instance in Fig. 5). Different values of the parameter $n$ are used over the range 1 to 4 in order to reproduce either the exponential law or the de Vaucouleurs' law. The standard deviation of the best fit is attached to a given curve of growth. 


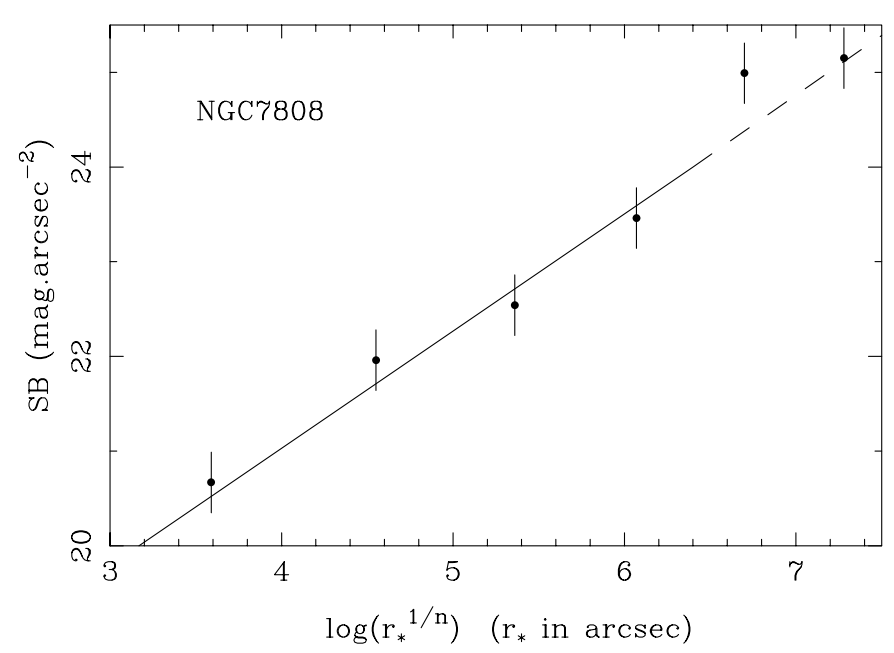

Fig. 5. Fit of a Sersic function to the brightness profile of a typical galaxy (NGC 7808). The error bars result from the standard deviation of the fit.

The galaxy profile is calculated as follows: Let $f_{i}\left(f_{i}=\right.$ $10^{-0.4 m_{i}}, i=1$ to 8 ) be the fluxes within the eight ellipses of surface $s_{i}=\pi a_{i} b_{i}$. The surface brightness $B_{i}$ at an equivalent radius $^{3}$

$r_{*, i}=\sqrt{\frac{s_{i}+s_{i-1}}{2 \pi}}$

is expressed as:

$B_{i}=-2.5 \log \frac{f_{i}-f_{i-1}}{s_{i}-s_{i-1}}$.

We limited the calculation to realistic values of the surface brightness; i.e., up to 24,23 and $22 \mathrm{mag} \operatorname{arcsec}^{-2}$ for $I, J$ and $K_{\mathrm{s}}$, respectively.

Using the standard deviation (rms residuals) of the fit, we calculated the weight of each individual magnitude as the inverse of the square of the standard deviation $\left(w=1 / \sigma^{2}\right)$. Then, we calculated the weighted mean magnitudes within the eight homothetic ellipses and their actual error (Paturel et al. 1997). This actual error takes into account both the internal uncertainty given by the standard deviation of the fit and the external uncertainty resulting from the comparison of independent measurements. An object is rejected if, at a given level, the magnitude cannot be calculated. Further, when the standard deviation of the fit is larger than a given limit, all measurements are also rejected. The adopted limits are: $0.6,0.8$ and $1.0 \mathrm{mag}$ for $I, J$ and $K_{\mathrm{s}}$, respectively.

We calculated the mean of each flag. The integer division gives the final flags, each being 0 or 1 . We also calculated the confidence in the recognition of an object as a galaxy. Many objects are confirmed galaxies. Hence, this parameter is useful only for objects not yet confirmed. This parameter is defined in magnitude as:

$G=-2.5 \log f_{\text {maxi }}+2.5 \log S_{\text {central }}$,

\footnotetext{
3 The equivalent radius is defined as $r_{*}=\sqrt{s / \pi}$. Note that the radius we use shares the elliptical ring in two equal areas.
}

the higher the parameter, the more secure the acceptation as galaxy. This parameter was used in our first attempt to extract galaxies from binned DENIS images (Vauglin et al. 1999). $f_{\text {maxi }}$ is the central peak flux (calibrated and corrected for extinction) and $S_{\text {central }}$ is the surface area of the central region (see previous section). The justification is that, unlike to a galaxy, a star has a small surface area and a high central intensity. Then, a typical star correspond to a small " $G$ ". On the contrary, a galaxy has a high " $G$ ". In order to test the significance of this parameter we show its distribution for two classes of objects (Fig. 6): confirmed and not confirmed galaxies, the latter ones being chosen near the galactic plane $\left(|b|<10^{\circ}\right)$ to increase the chance of having non extragalactic objects. For confirmed galaxies $G$ may be as low as 21, 20 or 18.5 in $I, J$ and $K_{\mathrm{s}}$, respectively. Below these values the classification as "galaxy" is uncertain. To be more conservative, it is better to accept unconfirmed object as galaxies when $G$ is larger than $22.5,21.0$ or 19.6 in $I, J$ and $K_{\mathrm{s}}$, respectively. In our catalog, the object type will be noted "G" for confirmed galaxies, "g" for not confirmed galaxies with high " $G$ " and "?" for objects that should be confirmed either by a visual inspection or by redshift measurement because $G$ is too small (i.e. below the adopted limit). For such a classification only $I$ and $J$ are considered because $K_{\mathrm{s}}$ is clearly not discriminant (see Fig. 6).

We plot (Fig. 7) the fraction of not confirmed galaxies versus the default magnitude in $I$-band. The fraction is almost constant up to the completeness limit $\left(I_{\text {lim }} \approx 15.5 \mathrm{mag}\right)$ found in Sect. 8 , but rises quickly above this limit. This shows that much work is still needed to confirm the extragalactic nature of many objects in LEDA. Nevertheless, the DENIS magnitudes will be available from this paper, when these objects will be confirmed.

\section{Magnitudes and diameters}

The largest ellipses used for flux integration are very large (up to twice the $D_{25}$ blue diameter). They are used to correct for the local variations of the sky background. The local brightness of the sky background is estimated as:

$B_{\mathrm{bg}}=\frac{f_{8}-f_{6}}{s_{8}-s_{6}}$

where $f_{i}$ is the flux within the $i$ th homothetic ellipse (i.e., $10^{-0.4 m_{i}}$ ) and $s_{i}$ its corresponding surface area. The corrected magnitude within the $i$ th ellipse is calculated as:

$m_{t}=-2.5 \log \left(f_{i}-s_{i} * B_{\mathrm{bg}}\right)$.

Actually, the corrected flux is calculated up to $6 / 8$ of twice the $D_{25}$ blue diameter (i.e., 1.5 times $D_{25}$ ). For the $K_{\mathrm{s}}$-band we integrated up to $D_{25}$ only and used the last four elliptical rings to estimate the local sky background. Now, we will consider only the corrected magnitudes.

We calculated four kinds of magnitude for each band:

1- Default magnitudes $I_{\mathrm{d}}, J_{\mathrm{d}}$ and $K_{\mathrm{d}}$. They are simply the integration of corrected flux up to the last ellipse $\left(1.5 D_{25}\right.$ for $I$ and $J, D_{25}$ for $K_{\mathrm{s}}$ ).

2- Total asymptotic magnitudes $I_{t}, J_{t}$ and $K_{t}$. They result from the extrapolation where the curve of growth becomes flat 

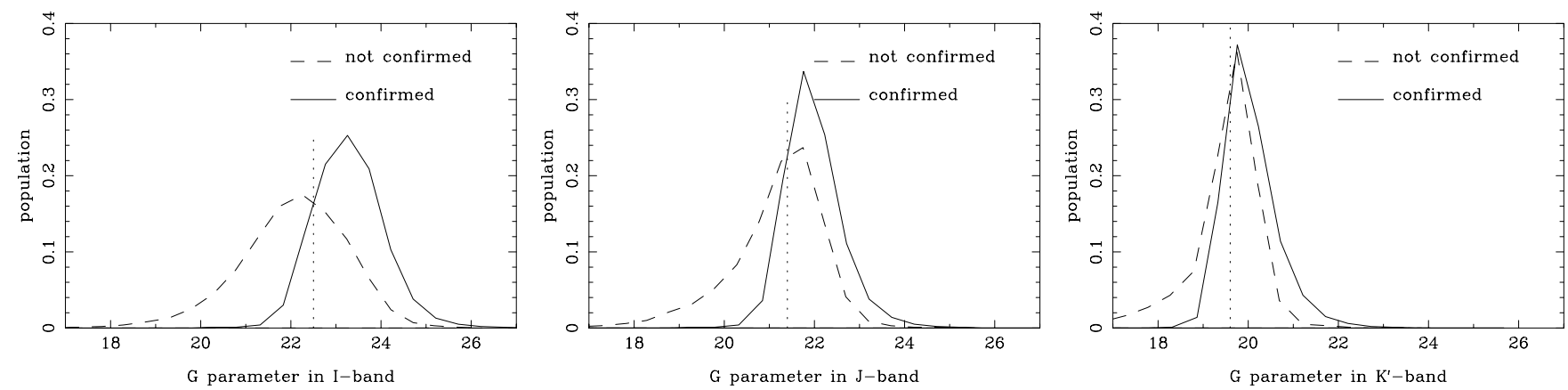

Fig. 6. Parameter $G$ for two populations: confirmed galaxies (full line) or unconfirmed objects near the galactic plane (dashed line). Below the limits drawn with a vertical doted line an unconfirmed object should not be considered as a galaxy without verification.

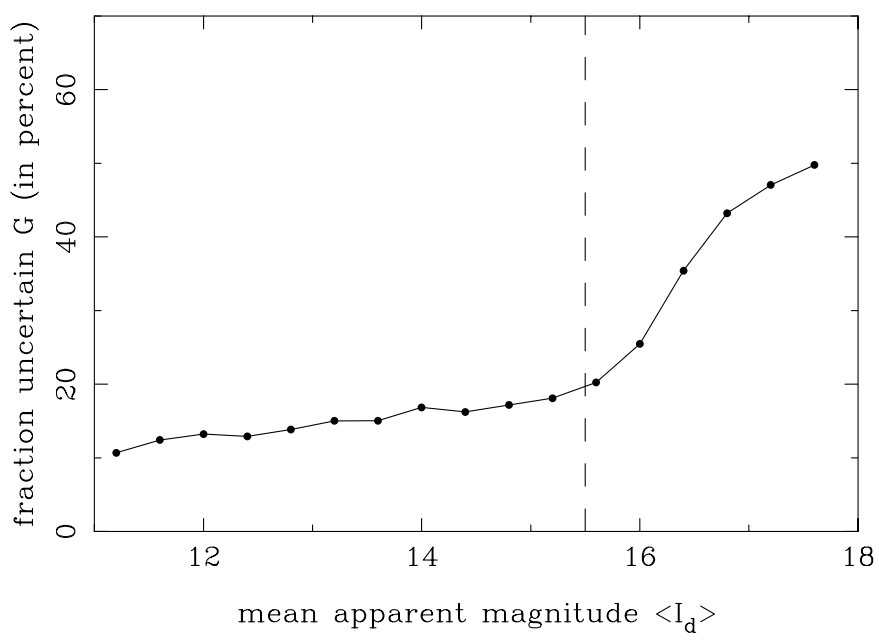

Fig. 7. Fraction (in percentage of the total number) of not confirmed galaxies versus the default magnitude, $I_{\mathrm{d}}$. The dashed line shows the completeness limit (dashed line) found in Sect. 8. Above this limit the fraction of not confirmed galaxies raises rapidly.

(i.e., where the derivative of the polynomial representation of the curve of growth is zero).

3- Kron magnitudes (Kron 1980) $I_{\mathrm{k}}, J_{\mathrm{k}}$ and $K_{\mathrm{k}}$. They result from the integration of corrected flux up to the adaptive equivalent Kron radius :

$r_{\mathrm{k}}=k \frac{\sum r f(r)}{\sum f(r)}$

where $f(r)$ is the flux up to the equivalent radius $r$. The calculation of $r_{k}$ is made up to the last ellipse. The balance between systematic and random errors is achieved for $k=2$ (Bertin \& Arnouts 1996).

4- Isophotal magnitudes $I_{\mathrm{s}}, J_{\mathrm{s}}$ and $K_{\mathrm{s}}$. They result from the integration of corrected flux up to the isophotal ellipse at $22.5 \mathrm{I} \mathrm{mag} \operatorname{arcsec}^{-2}, 21.0 \mathrm{~J} \mathrm{mag} \operatorname{arcsec}^{-2}$ and $20.0 K_{\mathrm{s}}$ mag $\operatorname{arcsec}^{-2}$, respectively.

The uncertainty on these magnitudes is calculated as the quadratic sum of the actual error on the surface brightness (calculated in previous section) and of the error on the surface area calculated as $1.086 \Delta s / s$, where $\Delta s$ is the uncertainty on the surface area, i.e. on the number of pixels. We estimated roughly this error as $\sqrt{s}$. So, the total error on total magnitudes is calculated as:

$s_{\mathrm{t}}=\left(\sigma^{2}(m)+1.179 / s\right)^{1 / 2}$.

In order to remove exceedingly faint objects (in LEDA some galaxies reach 20th $B$-magnitude) we consider only objects for which the total magnitude is smaller than: 18,17 or 16 for $I$, $J$ and $K_{\mathrm{s}}$, respectively. With the calculation of magnitudes, we calculated "total", "Kron" and "isophotal" diameters for $I, J$ and $K_{\mathrm{s}}$.

We calculated also the colours $I-J, I-K_{\mathrm{s}}$ and $J-K_{\mathrm{s}}$ from $K$-magnitudes to reveal discrepant magnitudes. Indeed, when a magnitude is involved in two discrepant colors (differing from the mean at the $3 \sigma$ level) it is removed because it results from an uncertain measurement. The mean values are the following:

$$
\begin{array}{ll}
\langle I-J\rangle=1.12 & \sigma=0.41 \\
\left\langle I-K_{\mathrm{s}}\right\rangle=2.25 & \sigma=0.62 \\
\left\langle J-K_{\mathrm{s}}\right\rangle=1.10 & \sigma=0.61
\end{array}
$$

The distributions are shown in Fig. 8. Note that a better estimate of colors can be deduced from the difference of magnitudes within a same ellipse. The study of colors will be conducted in a separate paper.

\section{Comparison with independent surveys}

We first make an internal comparison of our "default", "Kron" and "isophotal" magnitudes with our "total" magnitude (Table 2). The scales agree very well and we will consider zero point shifts only. Except for the isophotal magnitude, they do not differ from total magnitude by more than a few percent.

In order to have an external estimate of the standard deviation of our DENIS magnitudes, we make a comparison of our $I$ magnitudes with the $I$-band ones of the survey by Mathewson et al. (1992). Our $J$ and $K_{\mathrm{s}}$ magnitudes are compared with the $J$ and $K_{\mathrm{s}}$ magnitudes of the 2MASS survey (Jarrett et al. 2002). For 2MASS we use the "total" magnitudes (Cols. 41, 44 and 47 in the 2MASS extended source catalog).

We first made a cross-identification with these surveys. Each galaxy of our catalog is searched in other catalogs within a radius of $r=10^{\prime \prime}$. The cross-identification is accepted when there is only one candidate within $r$ and when the agreement between coordinates is better than $5^{\prime \prime}$. 

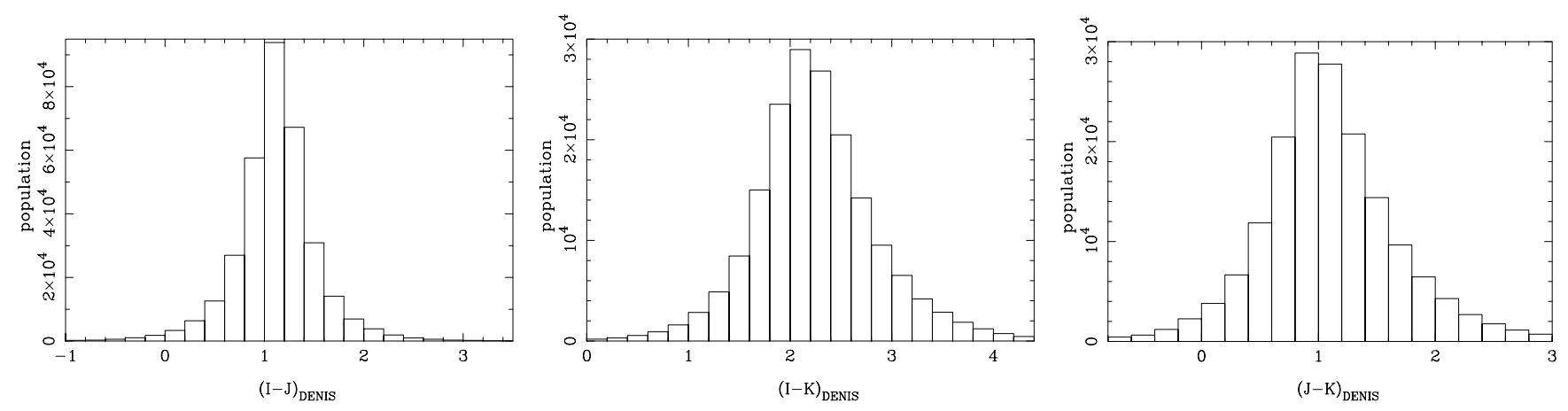

Fig. 8. Colours $I-J, I-K_{\mathrm{s}}$ and $J-K_{\mathrm{s}}$, calculated from total magnitudes.

Table 2. Comparison of zero-points of different kinds of apparent magnitude ("default", "Kron" and "isophotal") with the "total" magnitude.

\begin{tabular}{llrrr}
\hline \hline$X$-axis & $Y$-axis & $\langle X-Y\rangle$ & $\sigma$ & $n$ \\
\hline DENIS $I_{\mathrm{t}}$ & DENIS $I_{\mathrm{d}}$ & -0.058 & 0.043 & 93666 \\
DENIS $I_{\mathrm{t}}$ & DENIS $I_{\mathrm{k}}$ & -0.063 & 0.017 & 91811 \\
DENIS $I_{\mathrm{t}}$ & DENIS $I_{\mathrm{s}}$ & -0.214 & 0.101 & 81536 \\
DENIS $J_{\mathrm{t}}$ & DENIS $J_{\mathrm{d}}$ & -0.053 & 0.061 & 49746 \\
DENIS $J_{\mathrm{t}}$ & DENIS $J_{\mathrm{k}}$ & -0.068 & 0.027 & 49357 \\
DENIS $J_{\mathrm{t}}$ & DENIS $J_{\mathrm{s}}$ & -0.304 & 0.121 & 39175 \\
DENIS $K_{\mathrm{t}}$ & DENIS $K_{\mathrm{d}}$ & -0.025 & 0.044 & 19406 \\
DENIS $K_{\mathrm{t}}$ & DENIS $K_{\mathrm{k}}$ & -0.136 & 0.086 & 20217 \\
DENIS $K_{\mathrm{t}}$ & DENIS $K_{\mathrm{s}}$ & -0.115 & 0.102 & 9923 \\
\hline
\end{tabular}

A cut at a limiting magnitude $\left(16,15\right.$ and 14 for $I, J$ and $K_{\mathrm{s}}$, respectively) avoids the classical bias that distorts the relationship (Paturel et al. 1991) when the catalogs involved in the comparison have different depths. The effect of the choice of these limits is studied below (Table 4). Further, in order to obtain the nominal standard deviation, we first made the comparison for confirmed galaxies, with no flags and with an internal actual error less than $0.2 \mathrm{mag}$. This last limitation practically does not affect the results. Indeed, we repeated the calculation with an internal error as large as 0.9 mag without having a significant change in the final standard deviation (see Table 5). The case of less accurate objects (those with a flag) is studied in next section.

The different results of the two-by-two comparisons are summarized in Table 3. Examples of the comparison are given in Fig. 9 for the default magnitudes: $I_{\mathrm{d}}, J_{\mathrm{d}}$ and $K_{\mathrm{d}}$.

For the comparison with Mathewson's sample, we considered the direct regression (because Mathewson's magnitudes are assumed to be much more accurate than our magnitudes), while for the comparison with 2MASS we used the mean regression, because we assume that both systems have nearly the same accuracy (this is a first estimate, probably leading to an underestimation of our standard deviations). The slopes for $I$-band magnitudes are not significantly different from one. For $J$ and $K_{\mathrm{s}}$-bands, the small departure from one, always leads to a shift in magnitude smaller than half the standard deviation, over a range of $10 \mathrm{mag}$. Thus, we will provisionaly consider only the zero-point differences.
Table 3. Comparison of zero-points of our DENIS magnitudes, with those of other samples ("Mathewson" and "2MASS").

\begin{tabular}{llrrr}
\hline \hline$X$-axis & $Y$-axis & $\langle X-Y\rangle$ & $\sigma$ & $n$ \\
\hline Math. $I$ & DENIS $I_{\mathrm{d}}$ & 0.018 & 0.144 & 599 \\
Math. $I$ & DENIS $I_{\mathrm{t}}$ & 0.085 & 0.142 & 605 \\
Math. $I$ & DENIS $I_{\mathrm{k}}$ & 0.023 & 0.143 & 606 \\
Math. $I$ & DENIS $I_{\mathrm{s}}$ & 0.143 & 0.185 & 542 \\
2MASS $J$ & DENIS $J_{\mathrm{d}}$ & 0.031 & 0.242 & 48442 \\
2MASS $J$ & DENIS $J_{\mathrm{t}}$ & 0.089 & 0.218 & 49140 \\
2MASS $J$ & DENIS $J_{\mathrm{k}}$ & 0.023 & 0.210 & 48439 \\
2MASS $J$ & DENIS $J_{\mathrm{s}}$ & -0.210 & 0.214 & 38199 \\
2MASS $K$ & DENIS $K_{\mathrm{d}}$ & 0.039 & 0.402 & 23538 \\
2MASS $K$ & DENIS $K_{\mathrm{t}}$ & 0.091 & 0.379 & 21499 \\
2MASS $K$ & DENIS $K_{\mathrm{k}}$ & -0.069 & 0.350 & 21943 \\
2MASS $K$ & DENIS $K_{\mathrm{s}}$ & -0.096 & 0.362 & 10300 \\
\hline 2MASS $J$ & DENIS $J_{\mathrm{d}}$ & 0.031 & 0.242 & 48442 \\
2MASS $J$ & DENIS $J_{\mathrm{t}}$ & 0.089 & 0.218 & 49140 \\
2MASS $J$ & DENIS $J_{\mathrm{k}}$ & 0.023 & 0.210 & 48439 \\
2MASS $J$ & DENIS $J_{\mathrm{s}}$ & -0.210 & 0.214 & 38199 \\
\hline
\end{tabular}

The zero-point shift (Table 3) is reasonably small (less than a few percents) when considering "default" magnitudes. Our total magnitudes are brighter than those from other surveys, but the zero-point shifts never exceed $0.1 \mathrm{mag}$. In another paper we will make more detailed comparisons, in order to convert magnitudes to homogeneous systems.

To estimate the standard errors of our magnitudes we assumed that all the error in $I$-band comes from our own $I$-magnitudes and that the errors in $J$ and $K_{\mathrm{s}}$ are the same than those of 2MASS $J$ and $K_{\mathrm{s}}$ magnitudes. In other words, the standard deviation of the $I$-band regression gives directly the standard error on our $I$-magnitudes while the standard error on $J$ - and $K_{\mathrm{s}}$-magnitudes is obtained by dividing the standard deviation of the regression by $\sqrt{2}$ (same error on both axes). The results for different limiting magnitudes are summarized in Table 4. Let us note again that the standard errors on $J$ and $K_{\mathrm{s}}$ magnitudes of 2MASS are probably smaller than ours. Thus our standard errors may be underestimated.

A comparison of our $I$ magnitudes with $J$ ones from 2MASS gives a standard deviation $\sigma \approx 0.21$. Assuming again that the error on both axes is the same, one deduces that the uncertainty on our $I$-band magnitude is about $0.21 / \sqrt{2}$, i.e., $\sigma(I) \approx 0.15$. This is in good agreement with the value estimated from the comparison with Mathewson's sample around 
Table 4. Standard error on our magnitudes as a function of the limiting magnitudes.

\begin{tabular}{rrrrrrr}
\hline \hline$m_{\lim }$ & $\sigma(I)$ & $n$ & $\sigma(J)$ & $n$ & $\sigma\left(K_{\mathrm{s}}\right)$ & $n$ \\
\hline 10.00 & - & - & 0.09 & 143 & 0.11 & 470 \\
11.00 & 0.10 & 14 & 0.08 & 685 & 0.13 & 1766 \\
12.00 & 0.10 & 67 & 0.09 & 2804 & 0.16 & 5517 \\
13.00 & 0.11 & 240 & 0.10 & 9703 & 0.20 & 13790 \\
14.00 & 0.12 & 461 & 0.12 & 25470 & 0.25 & 21943 \\
15.00 & 0.14 & 586 & 0.15 & 48439 & 0.26 & 22959 \\
16.00 & 0.14 & 586 & 0.16 & 53750 & 0.26 & 22961 \\
\hline
\end{tabular}

Table 5. Standard error on total magnitudes as a function of the flag. The first two lines give the result when there is no flag but for actual error limited to either 0.2 mag or 0.9 mag.

\begin{tabular}{lrrrrrr}
\hline \hline Flag & $\sigma(I)$ & $n$ & $\sigma(J)$ & $n$ & $\sigma\left(K_{\mathrm{s}}\right)$ & $n$ \\
\hline $\begin{array}{l}\text { no flag } \\
\text { (a.e. } \leq 0.2)\end{array}$ & 0.14 & 606 & 0.15 & 48439 & 0.25 & 21943 \\
no flag & 0.15 & 713 & 0.16 & 148350 & 0.27 & 85380 \\
$\begin{array}{l}\text { (a.e. } \leq 0.9) \\
f_{\text {zp }}\end{array}$ & 0.14 & 44 & 0.16 & 4291 & 0.26 & 1679 \\
$f_{\text {qlt }}$ & 0.14 & 61 & 0.16 & 4595 & 0.30 & 2782 \\
$f_{\text {edge }}$ & 0.12 & 14 & 0.17 & 294 & 0.25 & 354 \\
$f_{*}$ & 0.24 & 91 & 0.28 & 4249 & 0.36 & 3776 \\
\hline
\end{tabular}

the same limit ( 0.14 at 15 th mag). These results are obtained after one cycle of $2 \sigma$ rejection. Without any rejection, they give larger standard errors. For instance, for limiting magnitudes 16, 15 and 14 in $I, J$ and $K_{\mathrm{s}}$ respectively, we obtain 0.20 (instead of 0.14 ), 0.18 (instead of 0.14 ) and 0.28 (instead of 0.25 ).

We compared our $I$-band diameters at the isophote 22.5 $I$-mag $\operatorname{arcsec}^{-2}$ with those measured by Mathewson at the isophote $23.5 \mathrm{I}$-mag $\operatorname{arcsec}^{-2}$. The comparison is shown in Fig. 10. The shift between both diameter systems is 0.066 with a standard deviation $\sigma=0.052$. This shows that, on average, the accuracy of these diameters is as good as, e.g. Nilson's diameters ( $\sigma=0.06$ according to Paturel et al. 1991).

We estimate the quantity $\partial \log D / \partial \mu \approx 0.07$ for the $I$-band around the brightness $\mu=23 \mathrm{mag} \operatorname{arcsec}^{-2}$. For comparison, in $B$-band, around $25 \mathrm{mag} \operatorname{arcsec}^{-2}$, this quantity is 0.09 , for a typical spiral galaxy (Fouqué \& Paturel 1985).

\section{Influence of flags}

Some measurements have a flag to say that they can be affected by a problem. Four flags are used: $f_{\text {zp }}$ quality of the zero-point, $f_{\text {qlt }}$ quality of the night, $f_{\text {edge }}$ object near the edge of the frame and $f_{*}$ contamination by superimposed object. The flag indicating that the coordinates do not agree with original ones is not considered because the corresponding objects have been removed.

The standard deviations, calculated as previously, are given in Table 5. The calculation is made for the limiting magnitudes 16,15 and 14 for $I, J$ and $K_{\mathrm{s}}$, respectively. The most severe influence comes from $f_{*}$ (star contamination).

\section{The catalog of raw data}

The three catalogs for $I-, J$ - and $K_{\mathrm{s}}$-band have been merged in one catalog arranged as follows. Four lines are given for each galaxy. The first line gives the parameters common to the three photometric bands; the next three lines give diameters and magnitudes for each photometric band. The detailed description is given below with an example in Table 6 .

\subsection{First line: Identification and common parameters}

Column 1: right ascension and declination for the equinox 2000 in hours, minutes, seconds and tenths, and in degrees, arcminutes and arcseconds respectively. These coordinates are those calculated from the $I$-band images;

Col. 2: the Principal Galaxy Catalog numbering (Paturel et al. 2003a);

Col. 3: alternate names following our previous hierarchy (Paturel et al. 2003a). Names that correspond to a multiple system are written in parenthesis. Names that do not agree with NED identification have been written with a question mark (?). Names that have been moved from one galaxy to another since the first PGC(1989) catalog have been written with an exclamation point (!);

Col. 4: label indicating that the object is present in the 2MASS extended source catalog;

Col. 5: apparent blue diameter $\log D_{25}$ in $\log$ scale $\left(D_{25}\right.$ in $0.1 \mathrm{arcmin})$ at the isophote $25 \mathrm{~B}$-mag $\operatorname{arcsec}^{-2}$;

Col. 6: axis ratio in $\log$ scale (log of major axis to minor axis) at the isophote $25 \mathrm{~B}$-mag $\operatorname{arcsec}^{-2}$;

Col. 7: adopted position angle in degrees measured from the North to the East. Its value covers the range $0^{\circ}-180^{\circ}$;

Col. 8: object nature: confirmed galaxies $(\mathrm{G})$, possible galaxies ( $\mathrm{g}$ ) or object to be confirmed (?).

\subsection{Next three lines: Global parameters}

Column 1: photometric band designation $\left(I, J\right.$ or $\left.K_{\mathrm{s}}\right)$;

Col. 2: right ascension and declination for the equinox 2000 in decimal hours and degrees;

Col. 3: discrepancy $d$ of the coordinates with respect to the LEDA coordinates. $d$ is measured in arcseconds;

Col. 4: parameter $G$ (see text) to characterize the nature of the object for not confirmed galaxies;

Col. 5: flags of quality. Each flag has a value 0 when there is no problem or 1 when one can suspect the data to be affected. The flags characterize the following features (in this order from the left to the right): flag 1 for quality of zero point, flag 2 for quality of the night, flag 3 for quality of the position of the object within the frame (edge effect), flag 4 for superimposed sources;

Col. 6: number of independent measurements;

Col. 7: decimal logarithm of apparent total diameter $\log D_{\mathrm{t}}\left(D_{\mathrm{t}}\right.$ in $\left.0.1^{\prime}\right)$;

Col. 8: decimal logarithm of apparent Kron diameter $\log D_{\mathrm{k}}$ $\left(D_{\mathrm{k}}\right.$ in $\left.0.1^{\prime}\right)$;

Col. 9: decimal logarithm of apparent isophotal diameter $\log D_{\mathrm{s}}\left(D_{\mathrm{s}}\right.$ in $\left.0.1^{\prime}\right)$, at isophote $22.5,21.0$ or $20.0 \mathrm{mag} \operatorname{arcsec}^{-2}$ for $I, J$ or $K_{\mathrm{s}}$, respectively; 
Table 6. Description of the structure of the catalog for each galaxy. In the electronic form available at CDS, only the numerical data are given (the designation of columns are given here only for clearness). Further, the four lines are all written on a single line to make the reading easier. The catalog is sorted in Right Ascension for the Equinox 2000.

\begin{tabular}{llllllll}
\hline \hline Coordinates & PGC number & Name & 2MASS & $\log D 25$ & $\log R 25$ & PA & $t$ \\
\hline J000332.2-104439 & PGC0000243 & NGC 7808 & 2MASS & 1.05 & 0.03 & 103 & $\mathrm{G}$ \\
\hline
\end{tabular}

\begin{tabular}{|c|c|c|c|c|c|c|c|c|c|c|c|c|c|c|}
\hline Band & Coordinates (2000) & $d$ & $G$ & Flags & $n$ & $\log D_{\mathrm{t}}$ & $\log D_{\mathrm{k}}$ & $\log D_{\mathrm{s}}$ & $s(\log D)$ & $m_{\mathrm{d}}$ & $m_{\mathrm{t}}$ & $m_{\mathrm{k}}$ & $m_{\mathrm{s}}$ & $s(\mathrm{~m})$ \\
\hline$\overline{i:}$ & $0.05894-10.7442$ & 2. & 23.64 & 0000 & 2 & 1.16 & 1.04 & 0.96 & 0.01 & 12.24 & 12.23 & 12.31 & 12.44 & 0.09 \\
\hline$J:$ & $0.05894-10.7445$ & 1 . & 22.66 & 0000 & 1 & 1.14 & 1.04 & 0.87 & 0.02 & 11.22 & 11.18 & 11.24 & 11.46 & 0.15 \\
\hline$K^{\prime}:$ & $0.05896-10.7443$ & 3. & 21.88 & 0000 & 1 & 0.97 & 0.88 & 0.91 & 9.99 & 10.38 & 10.32 & 10.36 & 10.34 & 0.24 \\
\hline
\end{tabular}
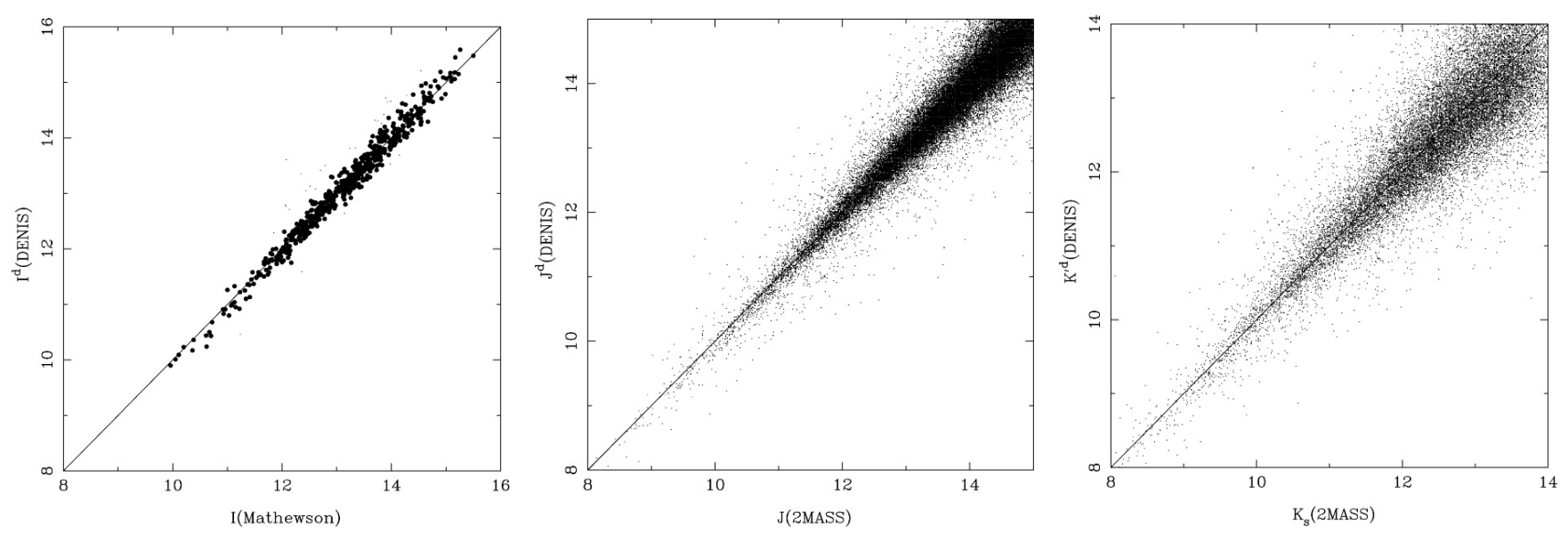

Fig. 9. Comparison of DENIS $I-, J-, K_{\mathrm{s}}$-bands "default" magnitudes with respectively the $I$-band magnitudes from Mathewson's sample and the $J$ and $K_{\mathrm{s}}$ magnitudes from the 2MASS survey.

Col. 10: standard deviation attached to the logarithms of diameters;

Col. 11: default apparent magnitude $m_{\mathrm{d}}$ integrated up to the last ellipse (see text);

Col. 12: apparent total magnitude $m_{\mathrm{t}}$, integrated up to the ellipse of major axis $D_{\mathrm{t}}$;

Col. 13: apparent Kron magnitude $m_{\mathrm{k}}$, integrated up to the ellipse of major axis $D_{\mathrm{k}}$;

Col. 14: apparent isophotal magnitude $m_{\mathrm{s}}$, integrated up to the ellipse of major axis $D_{\mathrm{s}}$;

Col. 15: standard deviation attached to the apparent magnitudes.

\subsection{Completeness of the catalog}

The catalog gives data for 753153 galaxies (among which there are 508224 galaxies, 34449 probable galaxies and 210480 galaxies to be confirmed). The catalog gives about (the figures may vary, depending on the considered magnitude or diameter): 668000 total I-band magnitudes, 576000 total $J$-band magnitudes, 357000 total $K_{\mathrm{s}}$-band magnitudes and 452000 total $I$-band diameters, 299000 total $J$-band diameters, 114000 total $K_{\mathrm{s}}$-band diameters.

The typical standard deviations on total magnitudes are $0.14,0.15$ and 0.25 , for $I-, J$ - and $K_{\mathrm{s}}$-band and limits in magnitude of 16,15 and 14 , respectively.

In order to estimate the mean completeness limit for each band, we plotted the $\log N\left(m<m_{\text {lim }}\right)$ vs. $m_{\text {lim }}$ (Fig. 11), for

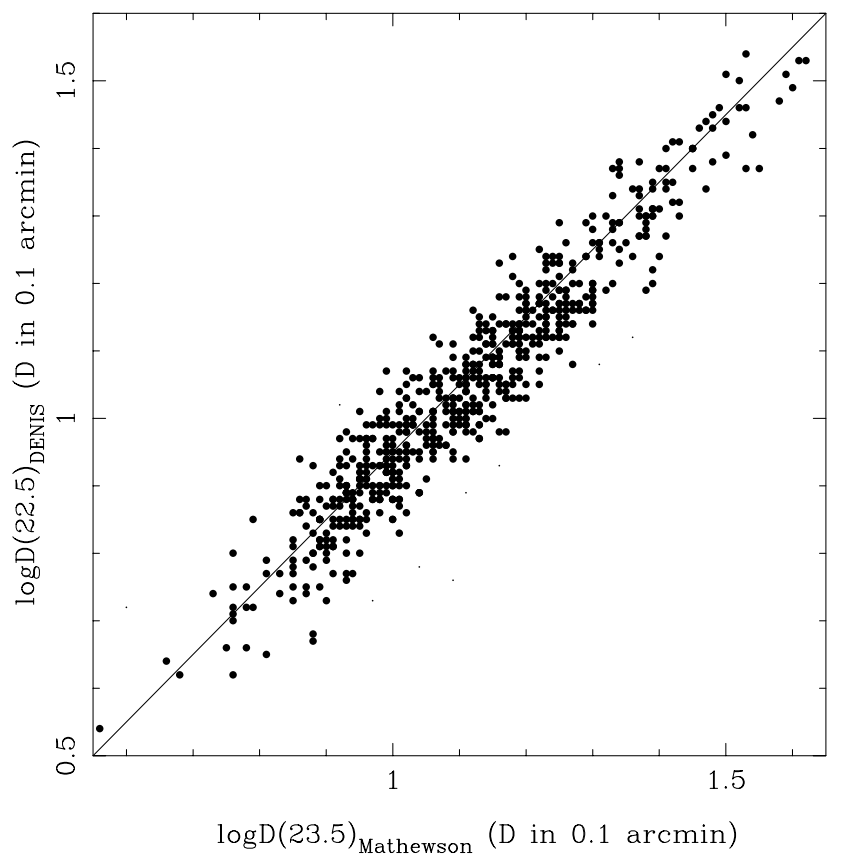

Fig. 10. Comparison of DENIS $I$-band diameter at the isophote $22.5 I-\mathrm{mag} \mathrm{arcsec}^{-2}$ with those measured by Mathewson at the isophote $23.5 I$-mag $\operatorname{arcsec}^{-2}$.

the three bands. The slopes differ significantly from the canonical value 0.6 , expected for a uniform distribution of galaxies. The slopes are: $0.52,0.54$ and 0.58 , for $I-, J-$ and $K_{\mathrm{s}}$-band 

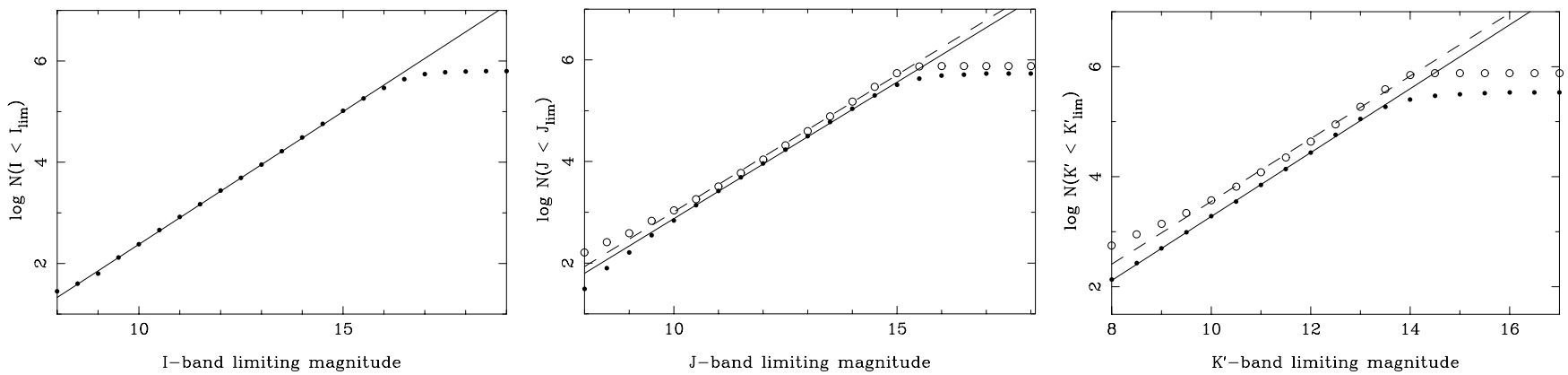

Fig. 11. Completeness curves for $I, J$ and $K_{\mathrm{s}}$ magnitudes. Black circles and solid line represent the completeness curves for the present DENIS catalog. Open circles and dashed line represent the completeness curves for the 2MASS extended source catalog.

respectively. This variation seems to be correlated with the uncertainty: the higher the uncertainty on magnitude, the closer to the canonical value 0.6 . This could be an illustration of the Eddington effect recently analyzed by Teerikorpi (2004).

From Fig. 11 one sees that the completeness limits are about : $15.5,14.5$ and 13.5 in $I, J$ and $K_{\mathrm{s}}$, respectively. For comparison we give the same plot for $J$ and $K_{\mathrm{s}}$ of the 2MASS extended source catalog (open circles), limited to the southern hemisphere. 2MASS is deeper in $K_{\mathrm{s}}(\approx 14 \mathrm{mag}$ instead of 13.5). In $J$, the deepness is almost the same for both survey. The 2MASS catalog has more objects than the DENIS one but it presents an excess of bright objects, while DENIS presents a lack of bright objects (in $J$ ). On the other hand, due to the selection of our objects from LEDA (essentially built from blue plates), our catalog is probably biased towards blue objects.

In conclusion, we want to warn any potential user that the contamination by superimposed objects (stars or companion galaxies) remains the major cause of problems. In using of individual measurements it is advisable to check that there is no contamination within $2 D_{25}$. This could be a way to improve the database in the future.

Acknowledgements. We thank all astronomers and technicians who participated to the measurements in La Silla. A part of the calculation (I-band) was made on a computer managed by Ph. Prugniel; we thank him for that. We thank the referee, T. H. Jarrett, for very good suggestions that helped us to improve the paper.

\section{References}

Bertin, E., \& Arnouts, S. 1996, A\&A, 117, 393

Borsenberger, J. 1997, in The Impact of Large-Scale Near-IR Surveys, ed. F. Garzon, et al. (Kluwer), 181

Epchtein, N., de Batz, B., Capoani, L., et al. 1997, The Messenger, 87, 27

Fouqué, P., \& Paturel, G. 1985, A\&A, 150, 192

Jarrett, T. H., Chester, T., Cutri, R., et al. 2000, AJ, 119, 2498

Kron, R. G. 1980, ApJS, 43, 305

Mathewson, D. S., Ford, V. L., \& Buchhorn, M. 1992, ApJ, 389, L5

Monet, D. B. A., Canzian, B., Dahn, C., et al. 1998, VizieR Online Data Catalog, 1252, 0

Paturel, G., Andernach, H., Bottinelli, L., et al. 1997, A\&AS, 124, 109

Paturel, G., Fang, Y., Petit, C., Garnier, R., \& Rousseau, J. 2000, A\&AS, 146, 19

Paturel, G., Fouqué, P., Buta, R., \& Garcia, A. M. 1991, A\&A, 243, 319

Paturel, G., Petit, C., Garnier, R., \& Prugniel, P. 1999, A\&AS, 140, 89 (Paper II)

Paturel, G., Petit, C., Prugniel, P., et al. 2003a, A\&A, 412, 45

Paturel, G., Petit, C., Rousseau, J., \& Vauglin, I. 2003b, A\&A, 405, 1

Rousseau, J., Paturel, G., Vauglin, I., et al. 2000, A\&A, 363, 62

Sersic, J. L. 1960, Atlas Austr. Cordoba Catalog

Vauglin, I., Paturel, G., Borsenberger, et al. 1999, A\&AS, 135, 133

Vauglin, I., Rousseau, J., Paturel, G., et al. 2002, A\&A, 387, 1

Teerikorpi, P. 2004, A\&A, 424, 73 